Supporting Information for:

\title{
Electron-Phonon Interaction Enables Strong Thermoelectric Seebeck Effect Variation in Hybrid Nanoscale Systems
}

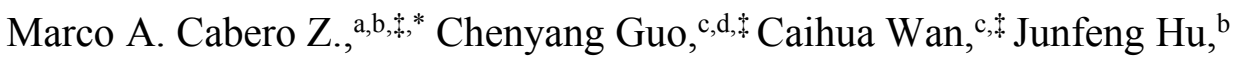 \\ Song Liu, ${ }^{\mathrm{a}}$ Mingkun Zhao, ${ }^{\mathrm{c}}$ Libo Zhang, ${ }^{\mathrm{a}}$ Qiuming Song, ${ }^{\mathrm{a}}$ Hanchen Wang, \\ Sa Tu, ${ }^{\mathrm{b}}$ Ningsheng Li, ${ }^{\mathrm{b}}$ Lutong Sheng, ${ }^{\mathrm{b}}$ Jilei Chen, ${ }^{\mathrm{b}}$ Yang Liu, ${ }^{\mathrm{b}}$ Bohang Wei, ${ }^{\mathrm{b}}$ \\ Jianyu Zhang, ${ }^{\mathrm{b}}$ Xiufeng Han,,${ }^{\mathrm{c}, \mathrm{d}}{ }^{*}$ Haiming $\mathrm{Yu},{ }^{\mathrm{b},{ }^{*}}$ and Dapeng $\mathrm{Yu}^{\mathrm{a}}$
}

a Shenzhen Institute for Quantum Science and Engineering, Southern University of Science and Technology, Shenzhen 518055, China.

${ }^{b}$ Fert Beijing Institute, MIIT Key Laboratory of Spintronics, School of Integrated Circuit Science and Engineering, Beihang University, Beijing, China.

${ }^{c}$ Beijing National Laboratory for Condensed Matter Physics, Institute of Physics, University of Chinese Academy of Sciences, Chinese Academy of Sciences, Beijing 100190, China.

${ }^{d}$ Center of Materials Science and Optoelectronics Engineering, University of Chinese Academy of Sciences, Beijing 100049, China.

$\$$ These authors contributed equally

* To whom correspondence should be addressed:

marco.cabero@buaa.edu.cn, xfhan@iphy.ac.cn, haiming.yu@buaa.edu.cn 


\section{Content}

I. Boltzmann Transport Equation (BTE) derived from Fourier's law

II. Boundary treatments

III. Electron-phonon interactions in hybrid systems

IV. Thermal resistance mismatch

V. Ruling out the effect of $\nabla \mathrm{Tz}$

Figure S1

Figure S2

Figure S3

Figure S4

Table S1 


\section{Boltzmann Transport Equation (BTE) derived from Fourier's law}

In the heat flow analysis, the sequence of rapid and slow relaxation events at two characteristic scales of transient response, associated with electron and phonon-assisted dynamics respectively (the so-called biexponential decay), appear to be universal and were observed in various experiments. ${ }^{1,2}$ To remedy this situation, various models have been proposed, such as the phonon hydrodynamics model ${ }^{3,4}$ the Cattaneo-Vernotte model $(\mathrm{C}-\mathrm{V}),{ }^{5,6}$ and others. ${ }^{7,8}$ To tackle phonon transport in a wide range of spatial scales, the phonon Boltzmann equation is required. ${ }^{9,10}$ This is a promising method for simulating hydrodynamics based on the relations between the lattice Boltzmann equation and Navier-Stokes equation. ${ }^{9-11}$ As the phonon velocity is considered to be that of the group velocity, ${ }^{11-12}$ the phonon Boltzman equation can be written as:

$$
\frac{\partial f}{\partial t}+\mathbf{V}_{\mathrm{g}} \cdot \boldsymbol{\nabla} f=\mathrm{C}(f)
$$

that describes the advection, variation, and scattering of phonons. ${ }^{13}$ Its discrete form results in the phonon lattice Boltzmann equation: ${ }^{26}$

$$
e_{i}\left(\mathbf{x}+\mathbf{c}_{i} \Delta t, t+\Delta t\right)-e_{i}(\mathbf{x}, t)=-\frac{e_{i}(\mathbf{x}, t)-e_{i}^{\mathrm{eq}}(\mathbf{x}, t)}{\tau}
$$

To recover the macroscopic heat transport equation, Chapman-Enskog expansion is applied to solve equation (2) and the relation between the lattice and phonon group velocity is obtained:

$$
c=\sqrt{\frac{7}{15}} v_{\mathrm{g}}
$$

Through the Chapman-Enskog expansion, the solution of the phonon lattice Boltzmann equation (PBE) can be also recovered. The dynamics of the phonon lattice Boltzmann equation in the analysis of our model at the nanoscale and the recovery of the Fourier's law allows the study of thermoelectric phenomena in both macro and nanoscales. 


\section{Boundary treatments}

The treatment of isothermal boundary conditions for the phonon Boltzmann equation scheme is provided here. Moreover, a periodic temperature gradient boundary condition is introduced to deal with the situation where heat is transported in a region with a large length-width ratio (i.g. thin-film/hall bar structure). The steady-state of phonon transport in three hybrid systems (hs) is initially set at a temperature $\mathrm{T}=293 \mathrm{~K}$ and the steady-state profiles of temperature and heat flow distributions are obtained approximately 3 minutes after the Peltier elements are turned on. ${ }^{14}$

The top and bottom boundaries are open boundaries with a given external air temperature (293K). We consider no-slip conditions at the surfaces of the sample when Peltier elements are turned on. We set the lateral boundaries periodic to the temperature, making the temperatures equal on both boundaries at every value. Finally, the models apply temperature continuity conditions and heat flow at all interior boundaries. For a detailed reference on the simulation see refs 33 and 34 .

\section{III.Electron-phonon interactions in hybrid systems}

The electron-phonon interaction increases the electronic damping $\sigma$ in the substrate $/ \mathrm{CoFeB} / \mathrm{Pt}$ hybrid system $(h s)$, and is described as:

$$
\Gamma_{\mathrm{el}}^{\sigma}=\Gamma_{\mathrm{s}-\mathrm{d}}^{\sigma}+\Gamma_{\mathrm{el}-\mathrm{el}}^{\sigma}+\Gamma_{\mathrm{el}-\mathrm{ph}}^{\sigma} .
$$

Where the number of scattering per second (relaxation time) is given by:

$$
\frac{1}{\tau}=\frac{1}{\tau_{\text {phonon }}}+\frac{1}{\tau_{\text {imperfection }}}+\frac{1}{\tau_{\text {surface }}}+\frac{1}{\tau_{\text {electron }}}
$$

At the steady-state, phonons drift with a velocity that is usually the sound velocity, resulting in:

$$
\frac{1}{\ell}=\frac{1}{\ell_{\text {phonon }}}+\frac{1}{\ell_{\text {imperfection }}}+\frac{1}{\ell_{\text {surface }}}+\frac{1}{\ell_{\text {electron }}}
$$

Therefore, the equation of thermal conductivity yields: 


$$
\kappa=\frac{1}{3} C v \ell
$$

Which is usually applicable in the diffusive or ballistic regime. ${ }^{63}$

The electron-electron ${ }^{15-16}$ and electron-phonon ${ }^{17,18}$ interactions have gained an increased interest in the past two decades. For example, coherent surface phonons have been investigated utilizing time-resolved second harmonic generation. ${ }^{19-20}$ The anharmonicity of surface phonon modes was considered to be greater than that of bulk phonon modes. Additionally, a strong oscillatory variation in coupling strength was observed when the film thickness was reduced. Furthermore, theoretical studies of surface phonon line-widths were reported. ${ }^{21}$ The results showed that the electron-phonon interaction (phonon drag) in ferromagnetic semiconducting thin films based on the s-d interaction model was strong especially due to the hybridization of $\mathrm{Co}^{22}$ Other studies in thin-films showed that the crystal symmetry could be broken, and the anharmonicity of the surface could increase a factor of 2 or 3 compared to that of the bulk. ${ }^{23,24}$ With the increase of Coulomb interaction the energy of the system increases. Therefore, the effect of the electronphonon interaction should be accountable..$^{22}$

\section{Thermal resistance mismatch}

The thermal resistance mismatch at the interface can be caused by acoustic mismatch and the result can be constructive or destructive wave interference. ${ }^{25}$ The thermal resistance mismatch is defined by the Kapitza equation as:

$$
\rho=\frac{T_{1}-T_{2}}{\Theta_{12}^{\text {tot }}\left(T_{1}\right)-\Theta_{21}^{\text {tot }}\left(T_{2}\right)}
$$

Where the heat flow is represented by the terms $\Theta_{12}^{\text {tot }}\left(T_{1}\right)-\Theta_{21}^{\text {tot }}\left(T_{2}\right)$. As the system approaches equilibrium, the change of interfacial resistance becomes smaller, due to varying $\Delta T=T_{1}-T_{2}{ }^{25}$ 
leading the Kapitza equation to 0 . Therefore, in our $h s$ due to the enormous size difference between the substrate and the FM/HM structure, we exclude any thermal resistance mismatch as the reason for different Seebeck voltage $\left(V_{\mathrm{S}}\right)$ in the three $h s$, since the heat flows from media 1 (substrate) into media 2 (FM/HM structure) is small. See the next section for more details.

\section{Ruling out the effect of $\nabla T_{z}$}

To experimentally verify that the contribution of the out-of-plane $\nabla T_{\mathrm{z}}$ to the voltage generation between c-d points is nil, a series of experiments were performed. First, the $h s$ was placed on the top of two Peltier elements (see Figure S3a) and situated between four magnetic poles (see Figure S3b). Then, the Peltier elements were turned on and the in-plane magnetic field was generated in the $x$-direction to measure $V$. The field in the $x$-direction was turned off to generate another magnetic field but this time in the $y$-direction and once again measure $V$. The magnetic field in both cases was swept from negative to positive and vice-versa. The results are shown in Figure S3c. The results confirm the non-existence of a measurable $V$ due to $\nabla T_{\mathrm{z}}$. Therefore, we conclude that the thermoelectromagnetic effect due to the out-of-plane $\nabla T_{z}$ is nil. These experimental results confirm that $\nabla T_{\mathrm{z}}$ is small, which is consistent with our simulation results. 


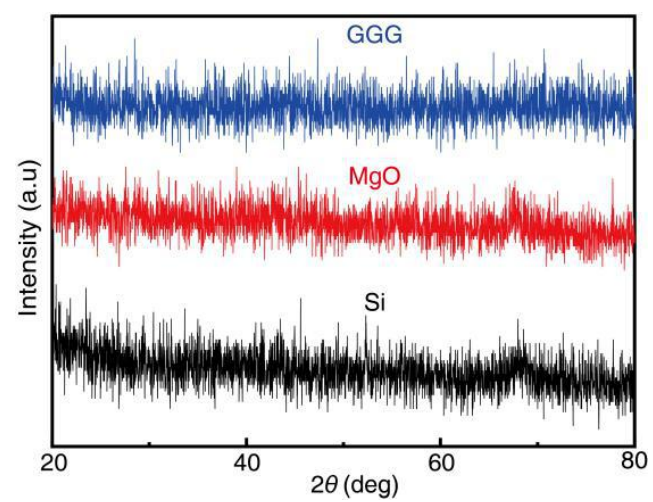

Figure S1. GIXRD patterns of three $h s$ subjected to $\mathrm{SiO}_{2}, \mathrm{MgO}$, and GGG substrates. The patterns confirm the amorphous structure of $\mathrm{CoFeB}$ in the three $h s$. 

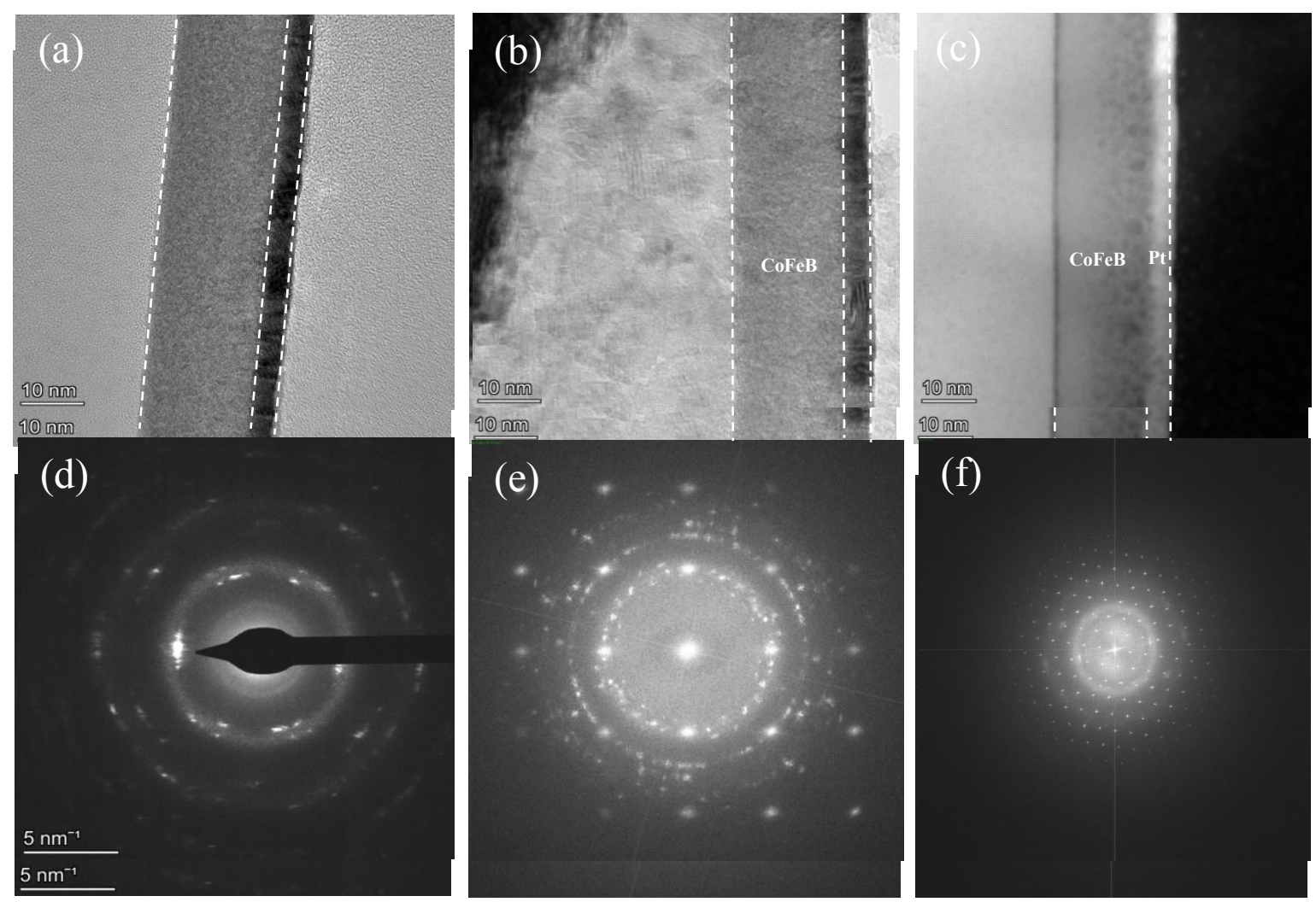

Figure S2. Structural characterization of three $h s$. a-f, The $h s$ subjected to $\mathrm{SiO}_{2}(\mathrm{a}, \mathrm{d}), \mathrm{MgO}(\mathrm{b}$, e), and GGG (c,f) substrates. a-c, TEM images were processed by Velox software v.2.10.0.71662027053d1 (https://www.fei-software-center.com/tem-apps/velox-download-page/). d-f, SAED patterns. 

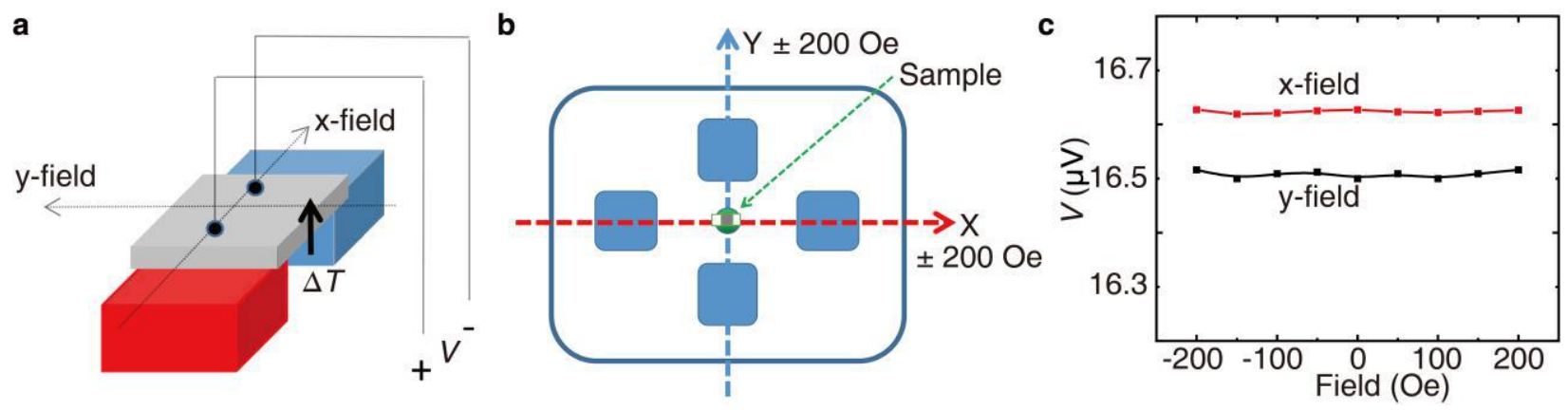

Figure S3. Ruling out the effect of $\nabla T_{\mathrm{z}}$. a, Schematic diagram of the device configuration for thermoelectromagnetic measurement. The hot and cold sides (red and blue cuboids) operate at $0.3 \mathrm{~W}$. b, Top view of the magnetic system configuration for the in-plane field application. The hs subjected to the $\mathrm{MgO}$ substrate was employed for the experiment. c, The voltage $V$ was measured three minutes after the Peltier elements were turned on. The magnetic field was first applied in the $x$-direction and then in the $y$-direction. The error of the measured $V$ was smaller than $1 \%$. These results confirm that the contribution of $\nabla T_{\mathrm{z}}$ to $V$ is nil. These results and the simulation results shown in the main document are in agreement. 

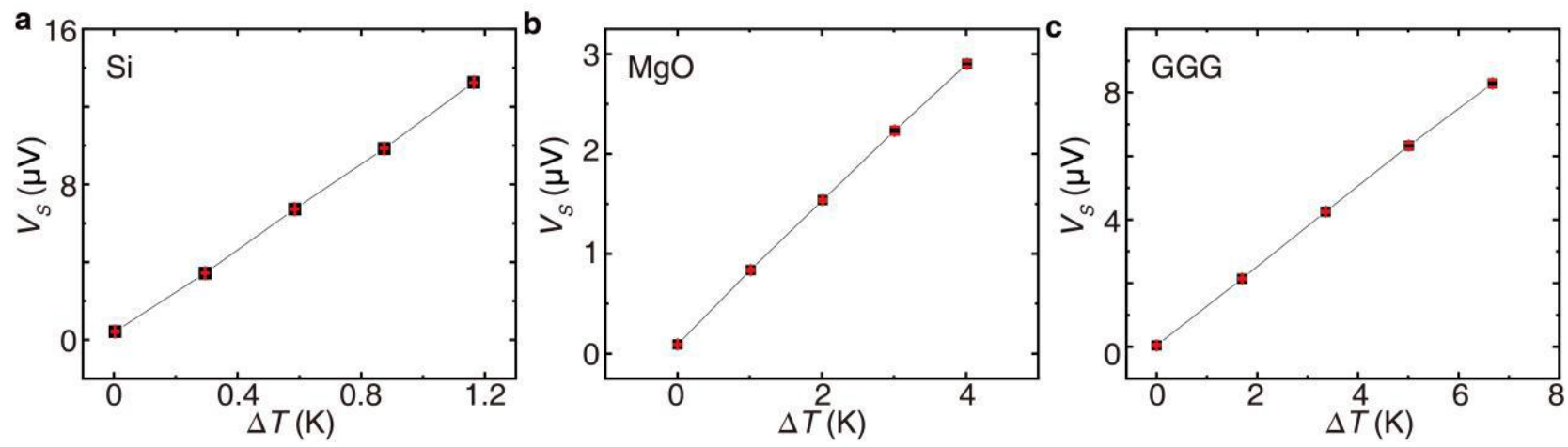

Figure S4. Seebeck coefficient measurement of three $h s$. a-c, Experimental results of twenty consecutive measurements of the Seebeck Voltage $V_{\mathrm{S}}$ as a function of $\Delta T$ in three $h s$ subjected to $\mathrm{SiO}_{2}$ (a), $\mathrm{MgO}$ (b), and GGG (c) substrates. The offset voltage at room temperature (when the Peltier elements were powered off) was smaller than $1 \%$. The error in the measurement of $V_{\mathrm{S}}$ was less than $2 \%$. The Seebeck coefficient of the connection wires $\left(S_{w} \approx 6.5 \mu \mathrm{V} \mathrm{K} \mathrm{K}^{-1}\right)$ was not considered for the calculation of $S$ shown in table 1 in the main document.

Table S1. Material parameters of the $h s$ used for the COMSOL simulation. The material parameters for the substrates were given by the manufacturer. *Other material parameters were obtained from [27], **[28-29], $* * *[30],{ }^{a}[31],{ }^{b}[32]$.

\begin{tabular}{|c|c|c|c|c|c|c|}
\hline Parameter & Unit & $\mathrm{SiO}_{2}$ & MgO & GGG & $\mathrm{CoFeB}^{\mathrm{a},{ }^{*}}$ & $\mathbf{P t}^{\mathbf{a}^{\mathrm{a}, * *}}$ \\
\hline $\begin{array}{c}\text { Thermal } \\
\text { conductivity }\end{array}$ & $\mathrm{W} / \mathrm{mK}$ & 1.4 & 59 & 7.05 & 87 & 70 \\
\hline & J/KgK & 1052 & 879 & 380 & 4490 & 132 \\
\hline $\begin{array}{c}\text { Density } \\
\text { Electrical } \\
\text { Conductivity }\end{array}$ & $\begin{array}{l}10^{3} \mathrm{Kg} / \mathrm{m}^{3} \\
10^{7} \Omega^{-1} \mathrm{~m}^{-1}\end{array}$ & 2.20 & 3.58 & 7.08 & $\begin{array}{l}8.22 \\
0.05^{\mathrm{b}}\end{array}$ & $\begin{array}{l}21.45 \\
0.4^{* * *}\end{array}$ \\
\hline
\end{tabular}




\section{References}

[1] Ulstrup, S.; Grubišić-Čabo, A.; Miwa, J. A.; Riley, J. M.; Grønborg, S. S.; Johannsen, J. C.; Cacho, C.; Alexander, O.; Chapman, R. T.; Springate, E. et al. Ultrafast Band Structure Control of a Two-Dimensional Heterostructure. ACS Nano 2016, 10, 6315.

[2] Pagliara, S.; Galimberti, G.; Mor, S.; Montagnese, M.; Ferrini, G.; Grandi, M. S.; Galinetto, P.; Parmigiani, F. Photoinduced $\pi-\pi^{*}$ Band Gap Renormalization in Graphite. J. Am. Chem. Soc. 2011, 133, 6318.

[3] Guo, Y.; Wang, M. Phonon Hydrodynamics and Its Applications in Nanoscale Heat Transport. Phys. Rep. 2015, 595, 1.

[4] Alvarez, F. X.; Jou, D.; Sellitto, A. Phonon Hydrodynamics and Phonon-Boundary Scattering in Nanosystems. J. Appl. Phys. 2009, 105, 014317.

[5] Cattaneo, C. Sulla Conduzione del Calore. Atti Semin. Mat. Fis. Univ. Modena 1948, 3, 83.

[6] Vernotte, P. Les Paradoxes de la Théorie Continue de l'équation de la Chaleur. Compt. Rendu. 1958, 246, 3154.

[7] Jou, D.; Lebon, G.; Criado-Sancho, M. Variational Principles for Thermal Transport in Nanosystems with Heat Slip Flow. Phys. Rev. E 2010, 82, 031128.

[8] Tzou, D. Y. Macro-to Microscale Heat Transfer: The Lagging Behavior; John Wiley \& Sons: New York, 2015.

[9] Chen, H.; Chen, S.; Matthaeus, W. H. Recovery of the Navier-Stokes Equations Using a Lattice-Gas Boltzmann Method. Phys. Rev. A 1992, 45, R5339(R).

[10] He, X.; Luo, L.-S. Theory of the Lattice Boltzmann Method: from the Boltzmann Equation to the Lattice Boltzmann Equation. Phys. Rev. E 1997, 56, 6811.

[11] Chen, S.; Doolen, G. D. Lattice Boltzmann Method for Fluid Flows. Annu. Rev. Fluid Mech. 1998, 30, 329.

[12] Escobar, R. A.; Ghai, S. S.; Jhon, M. S.; Amon, C. H. Multi-Length and Time Scale Thermal Transport Using the Lattice Boltzmann Method with Application to Electronics Cooling. Int. J. Heat Mass Transf. 2006, 49, 97.

[13] Peierls, R. E. Quantum Theory of Solids; Oxford University Press: Oxford, England, 1955.

[14] Guo, Y. Y.; Wang M. R. Lattice Boltzmann Modeling of Phonon Transport. J. Comp. Phys. 2016, 315, 1 .

[15] Quinn, J. J.; Ferrell, R. A. Electron Self-Energy Approach to Correlation in a Degenerate Electron Gas. Phys. Rev. 1958, 112, 812.

[16] Quinn, J. J. Range of Excited Electrons in Metals. Phys. Rev. 1962, 126, 1453. 
[17] McDougall, B. A.; Balasubramanian, T.; Jensen, E. Phonon Contribution to Quasiparticle Lifetimes in Cu Measured by Angle-Resolved Photoemission. Phys. Rev. B 1995, 51, 13891(R).

[18] Hellsing, B.; Eiguren, A.; Chulkov, E. V. Electron-Phonon Coupling at Metal Surfaces. $J$. Phys. Condens. Matter 2002, 14, 5959.

[19] Chang, Y.-M.; Xu, L.; Tom, H. W. K. Observation of Local-Interfacial Optical Phonons at Buried Interfaces Using Time-Resolved Second-Harmonic Generation. Phys. Rev. B 1999, 59, 12220 .

[20] Watanabe, K.; Dimitrov, D. T.; Takagi, N.; Matsumoto, Y. Coherent Surface Phonon at a GaAs (100) - c (8×2) Surface. Phys. Rev. B 2002, 65, 235328.

[21] Rahman, T. S.; Spangler, J. D.; Al-Rawi, A. Theoretical Studies of the Surface Phonon Linewidth. J. Phys. Condens. Matter 2002, 14, 5903.

[22] Wesselinowa, J. M. Electron-phonon Interaction in Ferromagnetic Semiconducting Thin Films. J. Phys. Condens. Matter 2015, 17, 507.

[23] Ma, S. K. S., De Wette, F. W.; Alldredge, G. P. Surface Relaxation and Thermal Expansion for the (001) Face of $\alpha$-Fe and Cu. Surf. Sci. 1978, 78, 598.

[24] Jayanthi, C. S.; Tosatti, E.; Pietronero, L. Surface Melting of Copper. Phys. Rev. B 1985, 31, 3456.

[25] Nazarenko, M.; Rosamond, M. C.; Gallant, A. J.; Kolosov, O. V.; Dubrovskii, V. G.; Zeze, D. A. A Simplified Model to Estimate Thermal Resistance Between Carbon Nanotube and Sample in Scanning Thermal Microscopy. J. Phys. D: Appl. Phys. 2017, 50, 494004.

[26] Magnetic Ultra-Thin Films, Multilayers, and Surfaces; Petroff, F.; Gijs, M. A. M. Eds.; Elsevier: Lausanne, Switzerland, 1997.

[27] Walter, M.; Walowski, J.; Zbarsky, V.; Münzenberg, M.; Schäfers, M.; Ebke, D.; Reiss, G.; Thomas, A.; Peretzki, P.; Seibt, M. et al. Seebeck Effect in Magnetic Tunnel Junctions. Nat. Mater. 2011, 10, 742 .

[28] Slack, G. A. Platinum as a Thermal Conductivity Standard. J. Appl. Phys. 1964, 35, 339.

[29] Kay \& Laby Online Version 1.0, 2005. Tables of Physical and Chemical Constants, 16th ed. 1995; www.kayelaby.npl.co.uk.

[30] Kockert, M.; Mitdank, R.; Zykov, A.; Kowarik, S.; Fischer, S. F. Absolute Seebeck Coefficient of Thin Platinum Films. J. Appl. Phys. 2019, 126, 105106.

[31] Lee, K.-D.; Kim, D.-J.; Lee, H. Y.; Kim, S.-H.; Lee, J.-H.; Lee, K.-M.; Jeong, J.-R.; Lee, K.-S.; Song, H.-S.; Sohn, J.-W. et al. Thermoelectric Signal Enhancement by Reconciling the Spin Seebeck and Anomalous Nernst Effects in Ferromagnet/Non-magnet Multilayers. Sci. Rep. 2015, 5, 10249. 
[32] Jen, S. U.; Yao, Y. D. Magnetic and Electric Properties of Amorphous CoFeB films. J. Appl. Phys. 2006, 99, 053701.

[33] Ortega, A. Air Cooling of Electronics: A Personal Perspective 1981 - 2001. In: Proc $18^{\text {th }}$ IEEE SMITHERM Symposium, 2002.

[34] Bailey, C. Modeling the Effect of Temperature on Product Reliability. In: Proc. $19^{\text {th }}$ IEEE SMITHERM Symposium, 2003.

[35] Huebener, R. P. Size Effect on Phonon Drag in Platinum. Phys. Rev. 1965, 140, A1834. 